\title{
Editorial
}

\section{CATARACT: THE MAIN CAUSE OF BLINDNESS IN LEPROSY}

World-wide in year 2000 there were approximately 20 million people blind from age-related cataract. ${ }^{1}$ Cataract is estimated to be responsible for at least $40 \%$, and of ten more, of all blindness. [Blindness is defined as a visual acuity (VA) of less than finger counting at $3 \mathrm{~m}$ $(<3 / 60)$ with the better eye; and severe visual impairment as a VA of less than finger counting at $6 \mathrm{~m}(<6 / 60)$ with the better eye.]

Cataract blindness is increasing, due to population growth and increasing longevity, combined with insufficient eye care services. Surveys have shown that $0 \cdot 7-1 \%$ of the population in developing countries suffer from blindness, of which $40-80 \%$ is due to cataract. Cataract blindness increases dramatically above the age of 50 years, and in developing countries, lens opacification seems to start about 10 years earlier than in industrialized countries. In India alone, 8.4 million people are thought to be blind or severely visually impaired from cataract.

Blinding eye complications, specifically due to leprosy, have become relatively uncommon since the introduction of multidrug therapy (MDT) for leprosy and with earlier detection of disease. Thus age-related cataract is now the single most important cause of blindness, even amongst leprosy patients. Courtright ${ }^{2}$ in 1997 estimated a total of 350,000-400,000 leprosy patients suffering from blindness, of which about half was due to non-leprosy causes, mainly cataract. In a multicentre study of 844 blind eyes in leprosy patients, nearly half of the blindness was directly or indirectly related to cataract, ${ }^{3}$ and in a retrospective study among 678 patients in Uganda $^{4} 1.3 \%$ were found to be blind, of which nearly half was directly related to leprosy. Cataract was the most common cause of blindness. In Nepal, among 260 newly diagnosed and untreated leprosy patients, 47 (18\%) had some lens opacity or were aphakic, making it the most common eye lesion seen, ${ }^{5}$ and in a recent 11 -year follow-up study from Korea, ${ }^{6} 87 \%$ of incident cases of blindness were due to cataract.

In addition to age-related cataract, steroid-induced cataracts may occur in leprosy patients on long-term steroids for recurrent reactions. Steroid treatment to prevent permanent nerve damage has priority and should continue in spite of cataract formation. Vision loss from cataract is reversible by surgery at a later stage. Secondary cataract may also develop in recurrent or chronic uveitis. In multibacillary (MB) patients with pinpoint pupils, centrally located, even small, lens opacities have a great impact on visual acuity. Age-related and secondary cataract due to steroids or chronic uveitis tend to become bilateral, and $\mathrm{MB}$ patients seem more prone to develop cataract than paucibacillary (PB) patients. ${ }^{7}$

Typically, patients with cataract are elderly and give a history of gradual and painless loss 
of vision. Mature cataract presents as a gray-white opacity behind the pupil. Immature but visually disturbing cataract, in particular posterior subcapsular cataract, cannot be diagnosed so easily, but can be recognized by obscuration of the red reflex observed with a direct ophthalmoscope. Assessment of visual acuity and pupillary reaction are essential. Impaired visual acuity with a 'grayish' pupil in an elderly person is not always due to cataract. Retinal and optic nerve diseases as well as chronic glaucoma and changes in refraction may be the cause of gradual loss of vision. It is important that leprosy staff recognize visual impairment in elderly patients on MDT and in elderly RFT patients in 'care after cure' programmes.

The only treatment for cataract is surgery. Indications for cataract surgery will vary with the setting. In leprosy, disability grade 2 is defined as 'cannot count fingers at a distance of 6 $\mathrm{m}(<6 / 60)$ '. This is the level of severe visual impairment. A visual acuity of $<6 / 60$ with the better eye is thus a definite indication for referral to the eye care services for diagnosis and if cataract is confirmed, cataract extraction. Referral alone, however, may not be sufficient, and transport and subsidy for surgery may need to be provided in order for the patient to obtain treatment.

Cataract surgery has undergone significant developments in recent years. An array of different techniques is now available, from simple, low tech ICCE with aphakic spectacles; to ECCE, with or without artificial lens implantation (IOL); to small incision, sutureless techniques of lens removal with IOL; and finally high-tech and costly phako-emulsification. All lead to sight restoration, if performed well. Cataract surgery with IOL implantation has definite advantages. The quality of vision is better and it avoids the problem of lost or broken aphakic spectacles and the optical problems of correction of unilateral aphakia. With modern implant surgery the requirement of the cataract to be 'mature' is no longer necessary. IOL surgery is of extra advantage for leprosy patients, who may have collapsed noses that will not easily hold heavy spectacles, or severely damaged hands, which cannot handle spectacles. If IOL surgery is not possible for whatever reason, then good quality aphakic spectacles $(\mathrm{S}+10)$ should be provided at the time of discharge, as an integral part of the treatment.

The great majority of cataracts in leprosy patients are age related and in such patients the result of cataract surgery with IOL is assumed to be equal to surgery in non-leprosy patients. Intra-ocular involvement due to leprosy may be seen in elderly patients with long-standing MB disease. Small, distorted and non-dilating pupils, iris atrophy, posterior synechiae, anterior staphyloma, possibly complicated by corneal anaesthesia and corneal scarring from exposure keratitis, will make cataract surgery more challenging and the prognosis more guarded. A history of uveitis is associated with more intra-and postoperative complications, and the risks and benefits of surgery in such patients need to be considered. Experienced surgeons should operate on patients with complicated cataract and postoperative control with intensive steroid treatment may be necessary.

Patients with complicated cataract comprise not more than 5-10\% of all leprosy cataracts, although this percentage depends on the setting, being less in a general leprosy programme, and more in a leprosy settlement where patients have a long history of disease and severe disabilities. No prospective studies have been published on the results of cataract surgery with IOL in patients, with or without intra-ocular involvement. Such studies, with a follow-up of at least 1 year, are needed. ${ }^{8}$

Eyes with lagophthalmos and lower lid laxity are more prone to chronic conjunctivitis. Patients with nose collapse may have blocked lacrimal sacs and chronic discharge. Any infection carries a risk of causing postoperative endophthalmitis, a disastrous complication of intra-ocular surgery. Such conditions must therefore be treated before cataract extraction can 
be undertaken. Eyes with active uveitis should be deferred until the inflammation is under control. Leprosy patients with anaesthetic limbs and blind from cataract cannot avoid trauma and wounds, and are therefore prone to develop ulcers. Surgeons may be reluctant to operate on such patients, because of fear for infection. They are thus in a vicious circle - they receive injuries because they cannot see, and because they have injuries, they cannot be operated on to improve their vision. Surgeons should be encouraged to operate once the wounds are 'clean' and granulating. Ideally, smear positive MB patients should be treated with MDT before cataract surgery, and any immune mediated reactions should be controlled first.

Where surgical facilities are available, there may be other barriers that have to be overcome before patients will accept cataract surgery. These can be summarized as: A, Awareness; B, 'bad services'; C, cost; and D, distance.

A. Patients or caregivers may not be aware of the possibility to restore eyesight in cases of cataract, or may consider themselves 'too old'. Health care staff may not be aware of poor vision in their patients.

B. The outcome of surgery or general care may have been disappointing in previous patients, and this will discourage new patients.

C. Costs are a major constraint, not only for surgery, medicines, admission and cost of food, but also because of travel cost and loss of income for the escort.

D. Distance to a surgical facility and lack of transport, and the unfamiliar environment in the city, may be significant barriers.

For leprosy patients, in addition, there is the stigma, which makes access to general health facilities of ten more difficult. Some eye departments are not willing to admit manifest leprosy patients. Cataract surgery for blind or severely visually impaired leprosy patients is an essential part of the prevention of disability. Leprosy programmes should therefore establish a collaborative agreement with the nearest eye-care unit for referral and surgery of such patients.

Severely disabled patients often cluster in leprosy settlements and are therefore easily accessible. Often little can be done, but cataract blindness can be cured. Programmes responsible for such patients can arrange for annual eye screening and surgery for those with visual loss due to cataract and other treatable conditions, such as severe lagophthalmos with exposure keratitis. Surgery in age-related cataract, in otherwise unaffected eyes, should preferably be with IOL implantation, provided that the expertise and necessary infrastructure are available. Surgery can be offered at the leprosy institution or settlements.

In conclusion, cataract has become the commonest cause of blindness in leprosy. Leprosy patients may experience the additional barrier of stigma and difficult access to the eye care services. The prevalence of cataract blindness among leprosy patients may therefore be higher then in the general population. Specific groups, such as those living in settlements, should be targeted for annual screening with arrangement for surgery. Otherwise, cataract surgery for leprosy patients should be part of regular POD services and preferably performed through the general eye care services.

\section{References}

${ }^{1}$ Foster A. Cataract - a global perspective: output, outcome and outlay. Eye, 1999; 13: 449-453.

2 Courtright P, Lewallen S. Ocular manifestations of leprosy. In: Johnson, Minassian, Weale (eds) The epidemiology of eye disease. Chapman \& Hall Medical 
${ }^{3}$ ffytche TJ. The prevalence of disabling ocular complications of leprosy: a global study. Ind J Lepr, 1998; 70: 49-59.

4 Waddel K, Saunderson PR. Is leprosy blindness avoidable? The effect of disease type, duration and treatment on eye damage from leprosy in Uganda. Br J Ophthalmol, 1995; 79: 250-256.

5 Lubbers WJ, Schipper A, Hogeweg M, de Soldenhoff R. Eye disease in newly diagnosed leprosy patients in eastern Nepal. Lepr Rev, 1994; 65: 231-238.

6 Lewallen S, Tungpakorn NC, Kim SH, Courtright P. Progression of eye disease in 'cured' leprosy patients: implications for understanding the pathophysiology of ocular disease and for addressing eye care needs. $\mathrm{Br} J$ Ophthalmol, 2000; 84: 817-821.

7 ffytche TJ. Residual sight threatening lesions in patients completing multidrug therapy and sulphone monotherapy. Lepr Rev, 1991; 62: 35-43.

8 Waddel K. Intraocular lens implantation for cataract following leprosy. Lepr Rev, 1999; 70: 74-75.

Consultant Ophthalmologist to Netherlands Leprosy Relief,

Margreet Hogeweg

Department of Ophthalmology,

Leiden University Medical Centre,

PO Box 9600 ,

2300 RC Leiden,

Netherlands 